\title{
OBSERVATIONS SUR LA VARIABILITÉ DE LA PRODUCTION DU COUVAIN DE MÂLES DANS LES COLONIES D'ABEILLES (Apis mellifica L.)
}

\author{
Beobachtungen über die Variabilität der Produktion von Drohnenbrut \\ im Bienenvolk (Apis mellifica L.)
}

\section{J. LOUVEAUX, J. MESQUIDA et J. FRESNAYE}

Station de Recherches sur l'Abeille et les Insectes sociaux, I. N. R. A., 91-Bures-sur-Yvette

Laboratoire de Recherches de la Chaire de Zoologie, I. N. R. A., E. N. S. A., 65, rue de St-Brieuc 35-Rennes

Station expérimentale d'Apiculture, Centre de Recherches d'Avignon, I. N. R. A., 84-Montfavet

\section{SUMMARY}

OBSERVATIONS OF THE VARIABILITY OF THE

PRODUCTION OF DRONE BROOD BY NONEYBEE COLONIES

During the years 1969, 1970 and 1971 observations concerning drone production by honeybee colonies were made in Brittany and in Provence.

Amongst the six groups of colonies which were formed those for which drone production was non-existent or very small were studied. The genetic character of the reduction of drone production was proved. At least one parent born of an inbred rearing is present in the ancestry of queens which possess this character. A correlation between this fact and the genetic character was set up.

The theoretical and practical interest of selecting strains which produce either many or very few drones is discussed.

\section{RÉSUME}

Au cours des années 1969, 1970 et 1971, des observations ont été faites en Bretagne et en Provence sur la production des mâles par les colonies d'abeilles.

Parmi les six groupes de colonies qui ont été constitués, on a étudié plus particulièrement ceux pour lesquels la production de mâles était nulle ou très réduite. Le caractère génétique de la réduction de la production des mâles a été mis en évidence; une corrélation a été établie entre ce caractère et la présence dans l'ascendance des reines qui le présentent d'au moins un parent provenant d'un élevage consanguin.

On discute l'intérêt théorique et pratique de la sélection des souches qui produisent soit beaucoup soit très peu de mâles. 


\section{INTRODUCTION}

Toute colonie d'abeilles normale élève au cours de son cycle biologique annuel un certain nombre de mâles; les observations courantes faites dans la zone tempérée montrent que la production des mâles est toujours limitée dans le temps et très variable d'une colonie à l'autre.

Les recherches effectuées en vue de déterminer les facteurs physiologiques, écologiques, éthologiques et génétiques qui agissent sur cette production sont peu nombreuses. M. D. Allen (1958) a montré, en mesurant le couvain de 7 colonies d'abeilles, que la période de production de masse des mâles est limitée en Écosse à 4 à 5 mois (de mai à août ou septembre) et qu'il existe une variation annuelle ainsi qu'une variation individuelle importantes, certaines colonies élevant beaucoup moins de mâles que les autres. Poursuivant ses recherches, M. D. Allen (1963) montra que lorsqu'on fournit aux colonies des cadres bâtis en cellules de mâles, la production de mâles est significativement plus élevée et que, réciproquement, l'élimination systématique des bâtisses comportant des cellules de mâles réduit la production. Contrairement à l'opinion générale des apiculteurs qui considèrent que l'élevage d'un grand nombre de mâles diminue la production de miel, M. D. Alles n'a constaté aucune différence à ce point de vue entre les colonies; la production de miel et l'élevage du couvain d'ouvrières ne sont pas significativement différents chez les colonies qui élèvent beaucoup de mâles et chez celles qui en élèvent peu.

En 1965, M. D. Allen confirme et précise ses recherches antérieures; elle constate que la fourniture ad libitum de bâtisses en cellules de mâles ne conduit pas à une augmentation indéfinie de la production de mâles qui finit toujours par atteindre un plafond. En 1964, aucune colonie approvisionnée en cellules de mâles ad libitum n'a élevé plus de $2580 \mathrm{~cm}^{2}$ de couvain de mâles, soit la valeur de deux cadres complets. Par ailleurs, en dépit de l'influence très nette de la présence de nombreuses cellules de mâles sur la production des mâles, il existe une variabilité considérable à l'intérieur des groupes de colonies pour ce caractère. En particulier, l'auteur cite une colonie qui négligea complètement les rayons construits en cellules de mâles qui lui furent fournis; d'autres colonies ne montrèrent qu'une faible tendance à élever du couvain de mâles alors que d'autres se comportèrent de façon inverse. Aucun facteur (race, quantité de couvain d'ouvrières) n'a pu être associé clairement à cette variabilité.

Les recherches de $W_{\text {EISs }}(1962,1969)$ ont été faites dans le but de connaître la production ( normale » de couvain de mâles dans les colonies laissées à ellesmêmes de façon à éliminer toute influence des techniques apicoles. Dans ces conditions les 5 colonies de race carniolienne soumises à l'expérimentation ont construit entre 10 et $17 \%$ de cellules de mâles. Au moment du plus grand développement de la colonie les surfaces de couvain de mâles étaient comprises entre 7,5 et $18,7 \%$ (moyenne $14,1 \%$ ). 
Il faut encore signaler les observations de Michailov (1925), reprises par Gorbakov (1961). Ces auteurs ont découvert des colonies d'abeilles n'élevant pas du tout de mâles. Dans ce cas particulier c'est une anomalie anatomophysiologique de la reine qui la rend incapable de pondre des oeufs non fécondés. Le reflexe de fermeture du canal de la spermathèque au moment de la ponte dans les cellules de mâles faisait défaut.

Les observations que nous présentons ici ont été faites, au moins au départ, de façon tout-à-fait fortuite. Pour une étude sur les caractères biométriques d'abeilles hybrides de races géographiques, nous avions besoin d'échantillons de mâles, ce qui nous avait amenés à faire des prélèvements de couvain suffisamment mûr pour pouvoir éclore en étuve. Cette opération de routine se heurta en 1968 à un obstacle imprévu : certaines colonies n'élevaient pas de mâles ou en élevaient si peu qu'il était impossible de faire les prélèvements prévus. Nous fûmes done amenés, dès 1969 , à faire des observations plus précises sur ce phénomène et à les compléter en 1970 et 1971.

\section{MATÉRIEL ET MÉTHODES}

\section{A. - Les colonies d'abeilles}

Les observations ont été faites, d'une part dans un rucher situé au Rheu, à proximité de Rennes, en Bretagne, d'autre part à la Station expérimentale d'apiculture de l'I.N.R.A. à Montfavet (Vaucluse) en Provence.

Les ruches utilisées étaient du modèle Dadant-Blatt, 10 cadres. Aucune mesure n'a été prise, soit pour réduire, soit pour augmenter artificiellement la production des mâles. Les cadres ont toujours été garnis de feuilles de cire gaufrée complètes et normales (780 cellules au $\mathrm{dm}^{2}$ ). Les opérations de nourrissement ont toujours été limitées au strict minimum et n'ont jamais été faites en vue de stimuler la ponte de la reine.

Six groupes de colonies sont à considérer, les quatre premiers au Rheu, les deux derniers à Montfavet.

Groupe I. A l'origine, les quatre colonies de ce groupe avaient reçu en 1967 des reines sœurs obtenues la même année par élevage à la Station expérimentale d'apiculture. Du côté maternel, ces reines descendaient d'une souche italienne non consanguine, de race pure (Apis mellifica ligustica), importée d'Italie en 1965; leur mère avait été inséminée artificiellement par le sperme de mâles de race noire (Apis mellifica mellifica) appartenant à une sonche provençale ( $\left.N^{0} 415\right)$ conservée depuis 1961 à Montfavet en étroite consanguinité par insémination artificielle. Elles-mêmes avaient été fécondées au rucher de fécondation de Cavillargues (Gard) par les mâles locaux qui sont de race noire dans leur très grande majorité.

On peut donc considérer que les reines du groupe I étaient des reines hybrides présentant un génotype relativement homogène.

Groupe II. Les six colonies de ce groupe avaient été constituées en 1968 de la même façon que celles du groupe I. Toutefois, la souche provençale consanguine utilisée pour l'insémination artificielle était différente. Il s'agissait de la souche $\mathrm{N}^{\circ} 360$.

Groupe III. Ce groupe avait été constitué en 1968 à partir de reines sœurs de race noire appartenant à l'écotype provençal, élevées à la Station de Montfavet mais non consanguines. Elles avaient été fécondées au rucher de fécondation de Cavillargues avant leur expédition en Bretagne.

Les colonies des groupes I, II, III, ont été livrées à elles-mêmes et on les a laissées essaimer ou changer leur reine librement, ce qu'elles ont d'ailleurs fait dans plusieurs cas. On s'est contenté de noter les changements de générations. Toutes les reines ont été marquées.

Groupe IV. Ce groupe témoin comportant six colonies a été formé en 1968 avec des reines de race noire appartenant à une population bretonne locale. Elles étaient sœurs. Dans ce 
groupe, on n'a pas admis les renouvellements de reines spontanés, ceci en raison des risques de fécondations indésirables par les mâles hybrides étrangers présents dans le rucher. Les colonies ayant changé de reine ont été remplacées par d'autres ayant une reine de la souche bretonne utilisée au départ.

Groupe $V$. Ce groupe a été constitué à Montfavet en vue d'observations à effectuer en 1971. Il comportait sept colonies dont les reines, inséminées artificiellement, représentaient le croisement de deux souches provençales, $\mathrm{N}^{0 \text { s }} 207$ et 315 , conservées respectivement depuis 1963 et 1961 en étroite consanguinité par insémination artificielle. Il est intéressant de noter dès à présent que la souche $N^{\circ} 207$ n'a pas pu être conservée après 1971 ; elle ne produisait plus de mâles.

Groupe VI. Comme le précédent, ce groupe avait été constitué à Montfavet en 1971 mais, cette fois, avec cinq reines hybrides: de trois races. Ces reines étaient les filles de reines caucasiennes (Apis mellifica caucasica) importées d'U.R.S.S. et maintenues à l'état pur par insémination artificielle mais sans consanguinité étroite. Les mâles ayant servi à l'insémination artificielle des reines caucasiennes étaient fils de reines italiennes importées d'Italie et dont la souche était conservée par insémination artificielle sans consanguinité étroite.

Les reines du groupe VI, fécondées au rucher de fécondation de Cavillargues par des mâles provençaux étaient donc des hybrides multiples.

\section{B. - Les méthodes de mesure du couvain}

En 1969, nous nous proposions simplement d'estimer, colonie par colonie, l'importance de l'élevage des mâles au cours de la saison active. Nous n'avons pas fait de mesures; nous nous sommes contentés d'attribuer une note de 0 à 4 pour la surface du couvain de mâles au cours de chacune des 10 visites qui ont été faites. La note 0 correspondait à l'absence de couvain de mâles; la note 4, note maximum, indiquait que le couvain de mâles était abondant et présent sur plusieurs cadres.

En 1970 et 1971, nous avons adopté une méthode de mesure plus précise. D'une part les surfaces de couvain ont été mesurées selon la méthode de Fresnaye (1962), d'autre part les contours des surfaces occupées par le couvain de mâles ont été dessinés par transparence sur des feuilles rigides de chlorure de polyvinyle. Au laboratoire, on a ensuite déterminé ces surfaces au moyen d'un planimètre. En 1970, au Rheu, une seule mesure a été faite, le 23 juin. En 1971, les mesures ont été plus nombreuses : une par mois à partir de mars; à Montfavet on n'a pu faire en 1971 que deux séries de mesures, les 6 mai et 10 juin.

\section{C. - Calculs statistiques}

Pour l'analyse statistique des résultats nous avons procédé à la comparaison de quelques moyennes de surfaces de couvain en utilisant le test non paramètrique $H$ de KRUsKaLL et Wallis (1952). Les moyennes des groupes I, II et III ont été comparées à celles du groupe IV qui constitue un bon témoin.

\section{RÉSULTATS}

Les principaux résultats ont été regroupés dans le tableau 1 qui permet de faire un certain nombre de constatations.

1 - La grande variabilité de la production des mâles dans les colonies 


\begin{tabular}{|c|c|c|c|c|c|c|c|c|c|}
\hline & & 1967 & 1968 & \multicolumn{2}{|r|}{1969} & \multicolumn{2}{|r|}{1970} & \multicolumn{2}{|r|}{1971} \\
\hline $\begin{array}{l}\text { Groupe } \\
\text { Gruppe }\end{array}$ & 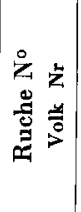 & 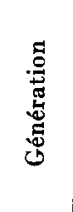 & 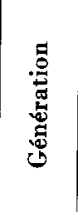 & 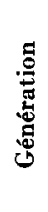 & 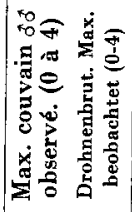 & 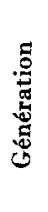 & 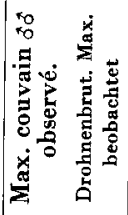 & 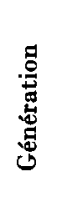 & 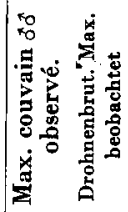 \\
\hline $\begin{array}{l}\text { I. Le Rheu. } \\
\qquad \frac{\text { ligustica }}{+\uparrow(\delta \delta \text { Pro. })} \times \frac{\delta d^{\circ} \text { P. } 415}{}\end{array}$ & $\begin{array}{l}15 \\
32 \\
22 \\
13\end{array}$ & $\begin{array}{l}1 \\
1 \\
1 \\
1\end{array}$ & $\begin{array}{l}2 \\
2 \\
1 \\
1\end{array}$ & $\begin{array}{l}2 \\
2 \\
1 \\
1\end{array}$ & $\begin{array}{l}2 \\
0 \\
0 \\
0\end{array}$ & $\begin{array}{l}2 \\
3 \\
1 \\
2\end{array}$ & $\begin{array}{l}7 \% \\
0 \% \\
0 \%\end{array}$ & $\begin{array}{l}2 \\
3 \\
1 \\
2\end{array}$ & $\begin{array}{l}6 \% \\
1 \% \\
0,1 \% \% \\
0 \%\end{array}$ \\
\hline $\begin{array}{l}\text { II. Le Rheu. } \\
\qquad \underbrace{}_{\text {o ligustica }} \times \underbrace{\star} \delta^{*} \text { Pro. }) \\
\end{array}$ & $\begin{array}{l}53 \\
65 \\
43 \\
58 \\
02 \\
03\end{array}$ & & $\begin{array}{l}1 \\
1 \\
1 \\
1 \\
1 \\
1\end{array}$ & $\begin{array}{l}1 \\
1 \\
1 \\
1 \\
1 \\
1\end{array}$ & $\begin{array}{l}2 \\
0 \\
3 \\
3 \\
1 \\
3\end{array}$ & $\begin{array}{l}1 \\
2 \\
1 \\
2 \\
2 \\
1\end{array}$ & $\begin{array}{l}0,8 \% \\
0,6 \% \\
0,1 \% \\
4 \% \\
-\overline{4} \%\end{array}$ & $\begin{array}{c}1 \\
2-3 \\
1 \\
2 \\
2 \\
2\end{array}$ & $\begin{array}{l}1 \% \\
0 \% \\
1 \% \\
4 \% \\
4 \% \\
4 \%\end{array}$ \\
\hline $\begin{array}{l}\text { III. Le Rheu. } \\
\text { 우 Pro. } \times \text { so Pro. }\end{array}$ & $\begin{array}{l}17 \\
41 \\
20 \\
19 \\
60 \\
40 \\
46 \\
68 \\
42\end{array}$ & & $\begin{array}{l}1 \\
1 \\
1 \\
1 \\
1 \\
1 \\
1 \\
1 \\
1\end{array}$ & $\begin{array}{l}1 \\
1 \\
1 \\
1 \\
1 \\
1 \\
1 \\
2 \\
1\end{array}$ & $\begin{array}{l}4 \\
4 \\
4 \\
4 \\
3 \\
4 \\
2 \\
4 \\
-\end{array}$ & $\begin{array}{l}2 \\
2 \\
1 \\
2 \\
2 \\
1 \\
2 \\
2 \\
2\end{array}$ & $\begin{array}{r}6 \% \\
3 \% \\
6 \% \\
11 \% \\
11 \% \\
8 \% \\
4 \% \\
12 \%\end{array}$ & $\begin{array}{c}2 \\
2 \\
1-2 \\
2 \\
2-3 \\
1-2 \\
2-3 \\
2 \\
2\end{array}$ & $\begin{array}{r}7 \% \\
12 \% \\
10 \% \\
7 \% \\
11 \% \\
14 \% \\
9 \% \\
11 \% \\
10 \%\end{array}$ \\
\hline $\begin{array}{l}\text { IV. Le Rheu. } \\
\qquad \text { \& } 502 \times \delta 0^{*} \text { Bre. }\end{array}$ & $\begin{array}{l}36 \\
37 \\
05 \\
39 \\
04 \\
29 \\
31 \\
55 \\
74\end{array}$ & & $\begin{array}{l}1 \\
1 \\
1 \\
1 \\
1 \\
1 \\
1\end{array}$ & $\begin{array}{l}1 \\
1 \\
1 \\
1 \\
1 \\
1\end{array}$ & $\begin{array}{l}4 \\
4 \\
4 \\
4 \\
4 \\
1\end{array}$ & $\begin{array}{l}\mathbf{1} \\
\mathbf{1} \\
\mathbf{1}\end{array}$ & $\begin{array}{r}6 \% \\
5 \% \\
10 \% \\
7 \% \\
6 \% \\
6 \%\end{array}$ & $\begin{array}{l}1 \\
1 \\
1\end{array}$ & $\begin{array}{r}9 \% \\
7 \% \\
12 \% \\
\\
14 \% \\
11 \% \\
11 \%\end{array}$ \\
\hline $\begin{array}{l}\text { V. Montfavet. } \\
\text { ๆ P. } 207 \times \text { ๙む P. } 315\end{array}$ & $\begin{array}{l}421 \\
376 \\
144 \\
366 \\
101 \\
444 \\
369\end{array}$ & & & & & & & $\begin{array}{l}1 \\
1 \\
1 \\
1 \\
1 \\
1 \\
1\end{array}$ & $\begin{array}{l}2 \% \\
4 \% \\
3 \% \\
4,5 \% \\
0,1 \% \\
0,1 \% \\
0,04 \%\end{array}$ \\
\hline $\begin{array}{l}\text { VI. Montfavet. } \\
\qquad \underbrace{}_{q \text { caucasica }} \times \frac{\delta \delta \text { ligustica }}{(\circlearrowleft \delta \text { Pro. })}\end{array}$ & $\begin{array}{l}430 \\
399 \\
253 \\
266 \\
249\end{array}$ & & & & & & & $\begin{array}{l}1 \\
1 \\
1 \\
1 \\
1 \\
1\end{array}$ & $\begin{array}{r}3,5 \% \\
7,9 \% \\
12,3 \% \\
10,7 \% \\
12,7 \%\end{array}$ \\
\hline
\end{tabular}

TABL. 1. - Récapitulation des observations effectuées entre 1967 et 1971

- En 1969, l'importance du couvain de mâles est exprimée par une note de 0 à 4 (Voir légende du tableau 5). La note qui figure dans la colonne est la note la plus élevée obtenue sur l'ensemble des 10 relevés effectués au cours de l'année.

- En 1970 et 1971 , les pourcentages qui figurent dans la colonne sont les pourcentages de couvain de mâles les plus élevés observés au cours de l'année (Pourcentages calculés par rapport à la surface totale du couvain).

- En ce qui concerne les générations on a adopté la convention suivante :

$1=$ reine d'origine,
$2=$ reine fille de la reine d'origine,
$3=$ reine petite-fille de la reine d'origine.

TAB. 1. - Zusammenstellung der Beobachtungen der Jahre 1967-1971

- 1969 : Die Ausdehnung der Drohnenbrut wird durch die Noten $0-4$ ausgedrückt (s. Legende zu Tab. 5). Die Note in der Spalte entspricht der höchsten Note, die bei 10 Kontrollen im Laufe des Jahres festgestellt wurde.

- 1970 und 1971 : Die Prozentangaben in der Spalte sind die höchsten Prozentsätze an Drohnenbrut, die im Verlaufe des Jahres beobachtet wurden (berechnet im Verhältnis zur gesamten Brutfläche).

- Die Generationen wurden wie folgt klassifiziert :

$1=$ Ursprungskönigin,

2 = Tochterkönigin der Ursprungskönigin,

$3=$ Enkelkönigin der Ursprunskönigin. 
d'abeilles se trouve nettement confirmée. Notons qu'il existe parmi les colonies étudiées des sujets qui n'élèvent pas ou pratiquement pas de mâles alors que d'autres ont une production ( normale » comparée à celle qui a été signalée par les différents auteurs.

2 - Au Rheu, la plus grande surface de couvain de mâles mesurée en 1970 le 23 juin, c'est-à-dire au moment où l'élevage peut être considéré comme étant, en principe, à son point culminant, est de $1314 \mathrm{~cm}^{2}$ (ruche No 40 , groupe III) (Tableau 2), et représente $11 \%$ de la surface totale du couvain. L'année suivante, c'est encore la même colonie qui fournit la plus grande surface de

\begin{tabular}{|c|c|c|c|c|c|}
\hline \multirow{2}{*}{$\begin{array}{c}\text { Groupe } \\
\text { Gruppe }\end{array}$} & \multirow{2}{*}{$\begin{array}{l}\text { Ruche No } \\
\text { Volk Nr }\end{array}$} & \multirow{2}{*}{ Génération } & \multicolumn{3}{|c|}{$\begin{array}{l}\text { Surface du couvain } \\
\text { Brut fläsche }\end{array}$} \\
\hline & & & $\stackrel{q}{\mathrm{~cm}^{2}}$ & $\begin{array}{c}a \\
\mathbf{c m}^{2}\end{array}$ & $\begin{array}{l}\sigma \\
\%\end{array}$ \\
\hline I. Hybrides 1967 & $\begin{array}{c}15 \\
32 \\
22 \\
13 \\
\text { Moy. }\end{array}$ & $\frac{2}{1} \frac{1}{2}$ & $\begin{array}{c}10482 \mathrm{~cm}^{2} \\
-\overline{5} \\
5720 \\
6240 \\
7480 \mathrm{NS}\end{array}$ & $\begin{array}{l}838 \mathrm{~cm}^{2} \\
\overline{0} \\
0 \\
270\end{array}$ & $\begin{array}{l}7,6 \% \\
\frac{0}{0} \\
2,3 \%\end{array}$ \\
\hline II. Hybrides 1968 & $\begin{array}{c}53 \\
65 \\
43 \\
58 \\
03 \\
\text { Moy. }\end{array}$ & $\begin{array}{l}1 \\
2 \\
1 \\
2 \\
1\end{array}$ & $\begin{aligned} 10394 \mathrm{~cm}^{2} \\
8785 \\
10753 \\
9552 \\
10241 \\
9945 \mathrm{NS} \\
\end{aligned}$ & $\begin{array}{r}86 \overline{\mathrm{cm}^{2}} \\
55 \\
7 \\
408 \\
39 \\
119 * * * \\
\end{array}$ & $\begin{array}{l}0,8 \% \\
0,6 \\
0,1 \\
4,1 \\
0,4 \\
1,3 \%\end{array}$ \\
\hline III. Provence 1968 & $\begin{array}{c}17 \\
41 \\
20 \\
19 \\
60 \\
40 \\
46 \\
68 \\
42 \\
\text { Moy. }\end{array}$ & $\begin{array}{l}2 \\
2 \\
1 \\
2 \\
2 \\
2 \\
1 \\
2 \\
2 \\
2\end{array}$ & $\begin{array}{rr}9 & 094 \mathbf{c m}^{2} \\
9 & 520 \\
10 & 330 \\
9 & \overline{864} \\
11 & 046 \\
9 & 011 \\
7 & 999 \\
8 & 180 \\
9 & 256^{*}\end{array}$ & $\begin{array}{c}54 \overline{\mathrm{cm}^{2}} \\
280 \\
630 \\
10 \\
1056 \\
1314 \\
789 \\
361 \\
1100 \\
760 \mathrm{NS}\end{array}$ & $\begin{array}{r}6 \% \\
3 \\
6 \\
11 \\
11 \\
11 \\
8 \\
4 \\
12 \\
8 \%\end{array}$ \\
\hline IV. Bretagne 1970 & $\begin{array}{c}36 \\
37 \\
05 \\
31 \\
55 \\
74 \\
\text { Moy. }\end{array}$ & $\begin{array}{l}1 \\
1 \\
1 \\
1 \\
1\end{array}$ & $\begin{array}{rr}10 & 483 \\
10 & 440 \\
9 & 802 \\
10 & 075 \\
9 & 562 \\
10 & 104 \\
10 & 078\end{array}$ & $\begin{array}{l}677 \mathrm{~cm}^{2} \\
600 \\
1038 \\
765 \\
598 \\
656 \\
722\end{array}$ & $\begin{array}{r}6 \% \\
5 \\
10 \\
7 \\
6 \\
6 \\
7 \%\end{array}$ \\
\hline
\end{tabular}

TABL. 2. - Surfaces de couvain (ouvrières et mâles) observées dans les groupes I à IV au Rheu le 23 juin 1970

Les tirets indiquent que la mesure de couvain n'a pas été possible. Le 0 indique une surface nulle.

* indique que la moyenne est significativement différente de celle du témoin (groupe IV) $P=0,10$ - ** $\mathbf{P}=0,05$ —** $P=0,01-\mathrm{NS}$, non significatif.

TAB. 2. - Brutfächen (Arbeiter und Drohnen) gemessen bei den Gruppen I - IV in Rheu am 23.6.1970

Die Gedankenstriche besagen, dass eine Messung nicht möglich war. Das Zeichen « 0 » bedeutet eine Oberfläche 0 .

* bedeutet dass sich das Mittel signifikant von dem der Kontrollgruppe IV unterscheidet $(P=0,10)$ - ** $\mathbf{P}=0,05-* * \mathbf{P}=0,01-\mathrm{NS}=$ nicht signifikant. 


\begin{tabular}{|c|c|c|c|c|c|c|}
\hline \multirow{3}{*}{$\underset{\substack{\infty \\
\text { J }}}{\Xi}$} & $\begin{array}{l}0 \\
\therefore\end{array}$ & 010101 & $|00|+||$ N $\mid$ & Imogentrame & \&: & \multirow{7}{*}{ 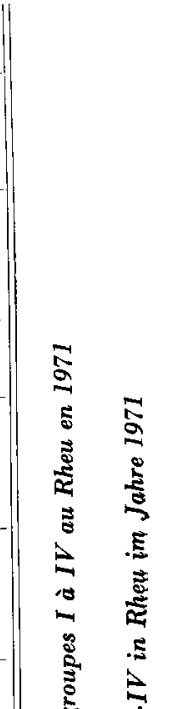 } \\
\hline & $\begin{array}{l}\tilde{g}_{0} \\
*_{0}\end{array}$ & 01010 & 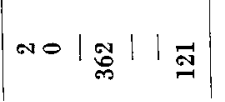 & $\mid$ | & ハ্লে & \\
\hline & $\begin{array}{c}\tilde{y} \\
x+1\end{array}$ & 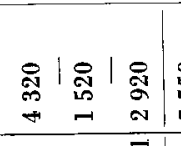 & 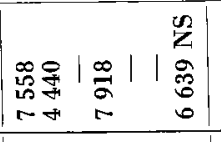 & 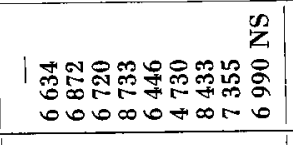 & 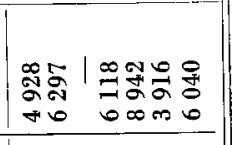 & \\
\hline \multirow{3}{*}{$\underset{\infty}{\infty}$} & $\begin{array}{l}2 \\
5 \\
5\end{array}$ & $\mid 1001$ & $\mid$\begin{tabular}{ll|}
0 & 0 \\
0 & 0
\end{tabular} & 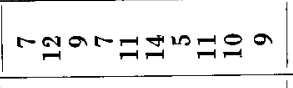 & an|こニニこ & \\
\hline & $\begin{array}{l}\pi \\
\vdots \\
0\end{array}$ & $|1-| r \mid$ & 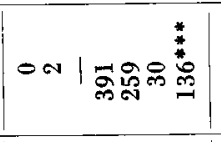 & 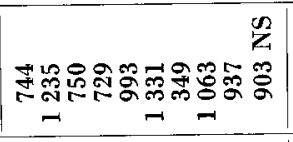 & 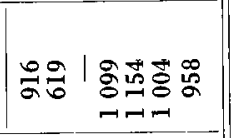 & \\
\hline & $\stackrel{\infty}{5}$ & 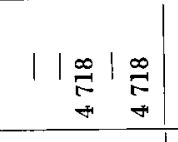 & 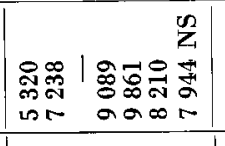 & 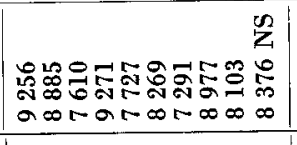 & 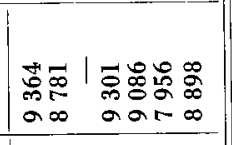 & \\
\hline \multirow{3}{*}{$\geq$} & $\begin{array}{l}0 \\
0 \\
\text { to }\end{array}$ & $0.00 \mathrm{a}$ & $\mid-11$ सकर्ष & noov|g|ano & 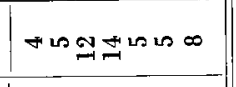 & \\
\hline & $\begin{array}{l}\tilde{j} \\
x_{0}\end{array}$ & 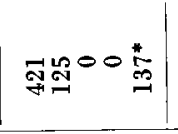 & 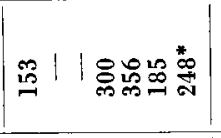 & 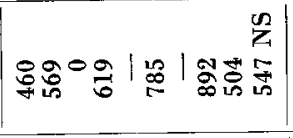 & 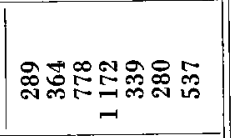 & 卷 \\
\hline & $\underset{0}{3}$ & 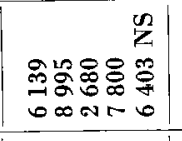 & 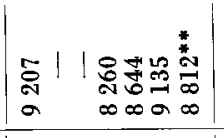 & 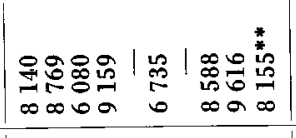 & 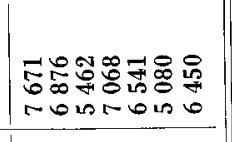 & ) \\
\hline \multirow{3}{*}{$\begin{array}{l}2 \\
2\end{array}$} & $\begin{array}{l}0 \\
0 \\
\text { ro }\end{array}$ & 00000 & OOnNot: & $\mid$ novornoso-o & $0000000 \mathrm{~m}$ & 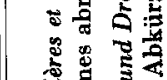 \\
\hline & $\begin{array}{l}\text { : } \\
\text { कo }\end{array}$ & $0000^{*}$ & 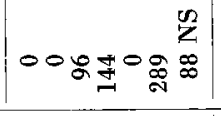 & 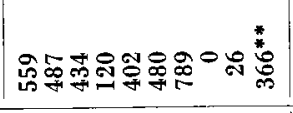 & $000000 \%$ & 竎 \\
\hline & $\underset{\infty}{\infty}$ & 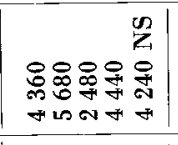 & 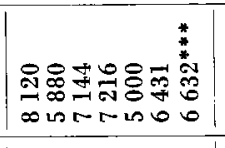 & 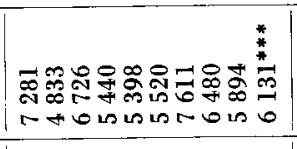 & 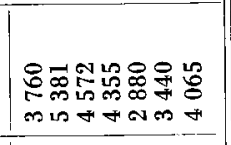 & 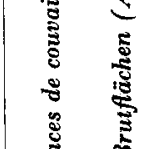 \\
\hline \multirow[b]{2}{*}{$\begin{array}{l}\Xi \\
\Xi\end{array}$} & $\begin{array}{l}\tilde{y} \\
x_{0} \\
\end{array}$ & 00000 & 0000000 & 0000000000 & 000 & $\begin{array}{c}\vec{n} \\
1 \\
1\end{array}$ \\
\hline & 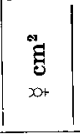 & 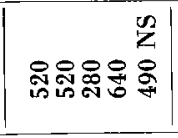 & | & 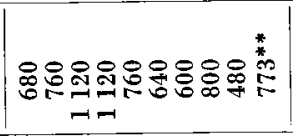 & 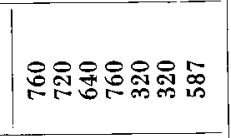 & 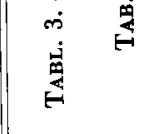 \\
\hline \multicolumn{2}{|c|}{ 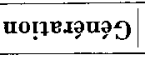 } & Nona & | ПNーNล⿻ & DNनNATANA & ルールローー & \\
\hline \multicolumn{2}{|c|}{ 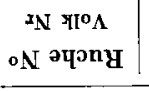 } & 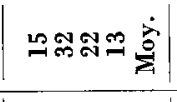 & 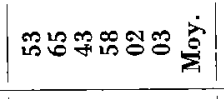 & 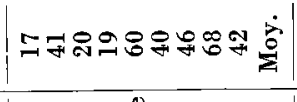 & 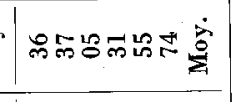 & \\
\hline \multicolumn{2}{|c|}{ 总总 } & 芴 & 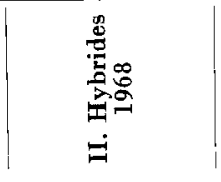 & 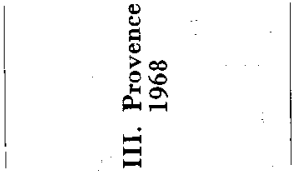 & 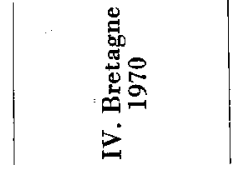 & \\
\hline
\end{tabular}


couvain de mâles le 28 juin avec $1331 \mathrm{~cm}^{2}$, soit $14 \%$ de la surface totale du couvain (Tableau 3).

A Montfavet, on a noté $2260 \mathrm{~cm}^{2}$ de couvain de mâles le 7 mai 1971 (ruche No 249 , groupe VI), cette surface ne représentant d'ailleurs que $12,7 \%$ du couvain total (Tableau 4).

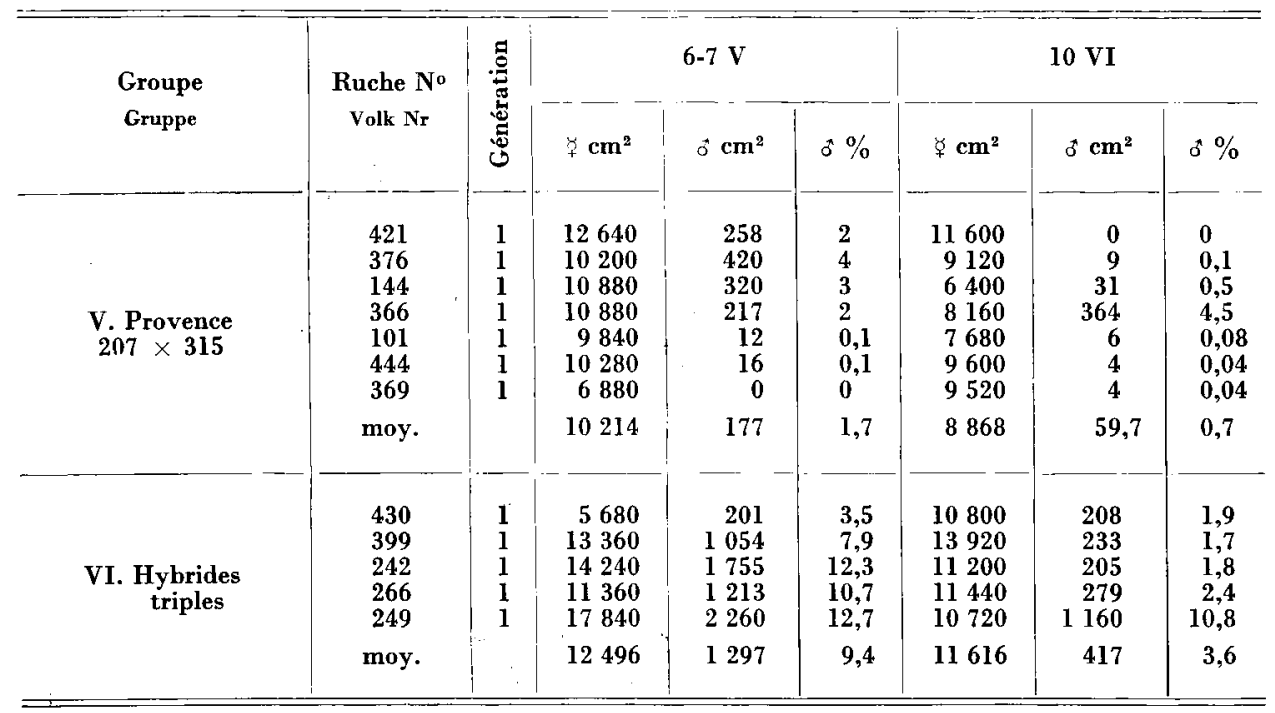

TABL. 4. - Surfaces de couvain (ouvrières et mâles) observées dans les groupes V et VI à Montfavet en 1971

TAB. 4. - Brutflächen (Arbeiter und Drohnen) bei den Gruppen V und VI in Montfavet im Jahre 1971

3 - La variation dans la production du couvain de mâles est nettement plus forte entre les groupes de ruches qu'à l'intérieur des groupes. Les différences entre certains groupes sont si grandes qu'une étude statistique paraît superflue.

Le groupe I comporte 3 colonies sur 4 qui ne donnent, sur 3 ans, que des quantités insignifiantes de couvain de mâles. La $4^{\mathrm{e}}$ a une production presque normale mais on notera qu'èlle est en seconde génération dès 1968 et que sa note de 1969 est basse puisqu'elle est de 2.

Le groupe II ne comporte que des colonies produisant peu ou pas de couvain de mâles et son comportement est sensiblement le même que celui du groupe I, quoique moins net.

Le groupe III peut être considéré comme « normal », surtout en 1969 et 1971. Il est très probable que pour ce groupe les mesures de 1970 effectuées le 23 juin ne sont pas situées exactement au moment du maximum. C'est le groupe qui, en moyenne, donne les plus fortes quantités de couvain mâle. 
Le groupe IV est très comparable au groupe III et donne, lui aussi, des résultats « normaux ». Les différences entre les groupes III et IV ne sont pas statistiquement significatives.

Le groupe $\mathrm{V}$ présente une assez bonne homogénéité dans la réduction de l'élevage du couvain de mâles. Trois colonies se comportent pratiquement comme des colonies sans mâles et ressemblent beaucoup à celles des groupes I et II.

Le groupe VI est, à nouveau, un groupe « normal » avec une seule colonie (No 430) se distinguant des autres; cette anomalie s'explique par le fait que la mesure de couvain du 7 mai 1971 a été faite peu de temps après un changement de reine.

\begin{tabular}{|c|c|c|c|c|c|c|c|c|c|c|c|}
\hline \multirow{2}{*}{$\begin{array}{l}\text { Groupe } \\
\text { Gruppe }\end{array}$} & \multirow{2}{*}{$\begin{array}{c}\text { Ruche } \mathrm{N}^{\circ} \\
\text { Volk Nr }\end{array}$} & \multicolumn{10}{|c|}{$\begin{array}{l}\text { Dates des observations } \\
\text { Datum der Beobachtungen }\end{array}$} \\
\hline & & $11 / 3$ & $15 / 4$ & $6 / 5$ & $\mid 20 / 5$ & $3 / 6$ & $24 / 6$ & $15 / 7$ & $29 / 8$ & $2 / 9$ & $15 / 10$ \\
\hline I. Hybrides & $\begin{array}{l}15 \\
32 \\
22 \\
13\end{array}$ & $\begin{array}{l}0 \\
0 \\
0 \\
0\end{array}$ & $\begin{array}{l}\mathbf{0} \\
0 \\
0 \\
0\end{array}$ & $\begin{array}{l}0 \\
0 \\
0 \\
0\end{array}$ & $\begin{array}{l}0 \\
0 \\
0 \\
0\end{array}$ & $\begin{array}{l}0 \\
0 \\
0 \\
0\end{array}$ & $\begin{array}{l}0 \\
0 \\
0 \\
0\end{array}$ & $\begin{array}{l}\mathbf{0} \\
\mathbf{0} \\
\mathbf{0} \\
\mathbf{0}\end{array}$ & $\begin{array}{l}2 \\
0 \\
0 \\
0\end{array}$ & $\begin{array}{l}0 \\
0 \\
0 \\
0\end{array}$ & $\begin{array}{l}0 \\
0 \\
0 \\
0\end{array}$ \\
\hline $\begin{array}{l}\text { II. Hybrides } \\
1968\end{array}$ & $\begin{array}{l}53 \\
65 \\
43 \\
58 \\
02 \\
03\end{array}$ & $\begin{array}{l}\mathbf{0} \\
\mathbf{0} \\
\mathbf{0} \\
\mathbf{0} \\
\mathbf{0} \\
\mathbf{0}\end{array}$ & $\begin{array}{l}0 \\
0 \\
1 \\
1 \\
0 \\
1\end{array}$ & $\begin{array}{l}\mathbf{2} \\
\mathbf{0} \\
\mathbf{3} \\
\mathbf{3} \\
1 \\
1\end{array}$ & $\begin{array}{l}0 \\
0 \\
1 \\
- \\
- \\
-\end{array}$ & $\begin{array}{l}0 \\
0 \\
0 \\
- \\
0 \\
0\end{array}$ & $\begin{array}{l}\mathbf{0} \\
0 \\
0 \\
0 \\
0 \\
3\end{array}$ & $\begin{array}{l}0 \\
0 \\
0 \\
0 \\
0 \\
3\end{array}$ & $\begin{array}{l}0 \\
0 \\
0 \\
0 \\
0 \\
0\end{array}$ & $\begin{array}{l}0 \\
0 \\
0 \\
0 \\
0 \\
0\end{array}$ & $\begin{array}{l}\mathbf{0} \\
\mathbf{0} \\
\mathbf{0} \\
\mathbf{0} \\
\mathbf{0} \\
\mathbf{0}\end{array}$ \\
\hline $\begin{array}{l}\text { III. Provence } \\
1968\end{array}$ & $\begin{array}{l}17 \\
41 \\
20 \\
19 \\
60 \\
40 \\
46 \\
68\end{array}$ & $\begin{array}{l}\mathbf{0} \\
0 \\
0 \\
0 \\
0 \\
0 \\
0 \\
0\end{array}$ & $\begin{array}{l}2 \\
0 \\
0 \\
1 \\
3 \\
0 \\
1 \\
2\end{array}$ & $\begin{array}{l}2 \\
1 \\
1 \\
2 \\
3 \\
2 \\
2 \\
3\end{array}$ & $\begin{array}{l}0 \\
0 \\
0 \\
0 \\
\\
0 \\
0 \\
-\end{array}$ & $\begin{array}{l}0 \\
0 \\
0 \\
0 \\
- \\
0 \\
0 \\
-\end{array}$ & $\begin{array}{l}4 \\
4 \\
4 \\
4 \\
-4 \\
1 \\
4\end{array}$ & $\begin{array}{l}\mathbf{0} \\
\mathbf{0} \\
\mathbf{3} \\
\mathbf{3} \\
\overline{3} \\
\mathbf{0} \\
\mathbf{3}\end{array}$ & $\begin{array}{l}0 \\
0 \\
2 \\
3 \\
0 \\
3 \\
0 \\
3\end{array}$ & $\begin{array}{l}0 \\
0 \\
0 \\
0 \\
0 \\
0 \\
0 \\
0\end{array}$ & $\begin{array}{l}0 \\
0 \\
0 \\
0 \\
0 \\
0 \\
0 \\
0\end{array}$ \\
\hline $\begin{array}{l}\text { IV. Bretagne } \\
1969\end{array}$ & $\begin{array}{l}36 \\
37 \\
05 \\
39 \\
04 \\
29\end{array}$ & $\begin{array}{l}0 \\
0 \\
0 \\
0 \\
0 \\
0\end{array}$ & $\begin{array}{l}\overline{2} \\
\overline{3} \\
2 \\
-\end{array}$ & $\begin{array}{l}- \\
2 \\
\overline{3} \\
2 \\
-\end{array}$ & $\begin{array}{l}- \\
\overline{-} \\
\overline{4} \\
\overline{-}\end{array}$ & $\begin{array}{l}- \\
- \\
- \\
- \\
- \\
-\end{array}$ & $\begin{array}{l}4 \\
4 \\
4 \\
4 \\
4 \\
0\end{array}$ & $\begin{array}{l}- \\
\overline{4} \\
4 \\
1 \\
1\end{array}$ & $\begin{array}{l}\overrightarrow{0} \\
0 \\
4 \\
0 \\
0\end{array}$ & $\begin{array}{l}0 \\
0 \\
0 \\
0 \\
0 \\
0\end{array}$ & $\begin{array}{l}0 \\
0 \\
0 \\
0 \\
0 \\
0\end{array}$ \\
\hline
\end{tabular}

TABL. 5. - Estimation des surfaces de couvain mâle des colonies des groupes I à IV au Rheu en 1969 Notation de 0 à 4 :

0 = pas de couvain de mâles,

$1=$ couvain de mâles en très faibles quantités,

$2=$ couvain de mâles par petites plaques isolées,

3 = couvain de mâles assez abondant sur quelques cadres,

4 - couvain de mâles très abondant sur plus de deux cadres,

- = observation non effectuée.

TAB. 5. - Schätzung der Drohenbrutflächen bei den Völkern der Gruppe I - IV in Rheu im Jahre 1969 Klassifizierung 0 - 4 :

$0=$ keine Drohnenbrut,

1 = ganz geringe Mengen Drohnenbrut,

$2=$ kleine isolierte Nester Drohnenzellen,

$3=$ ziemlich viel Drohnenbrut auf einigen Waben,

4 = sehr reichlich Drohnenbrut auf mehr als 2 Waben,

- = keine Beobachtung. 
4 - La courbe de la production du couvain de mâles au Rheu peut être étudiée avec une certaine précision sur les données de 1969 (Tableau 5) et de 1971 (Tableau 3).

En 1969 , le début de la période de production des mâles se situe après le 11 mars et avant le 15 avril. La fin de cette même période se place dans le courant du mois d'août. En 1971 les dates sont sensiblement les mêmes. On peut donc estimer qu'au Rheu la période d'élevage des mâles va du début d'avril jusque dans le courant du mois d'août.

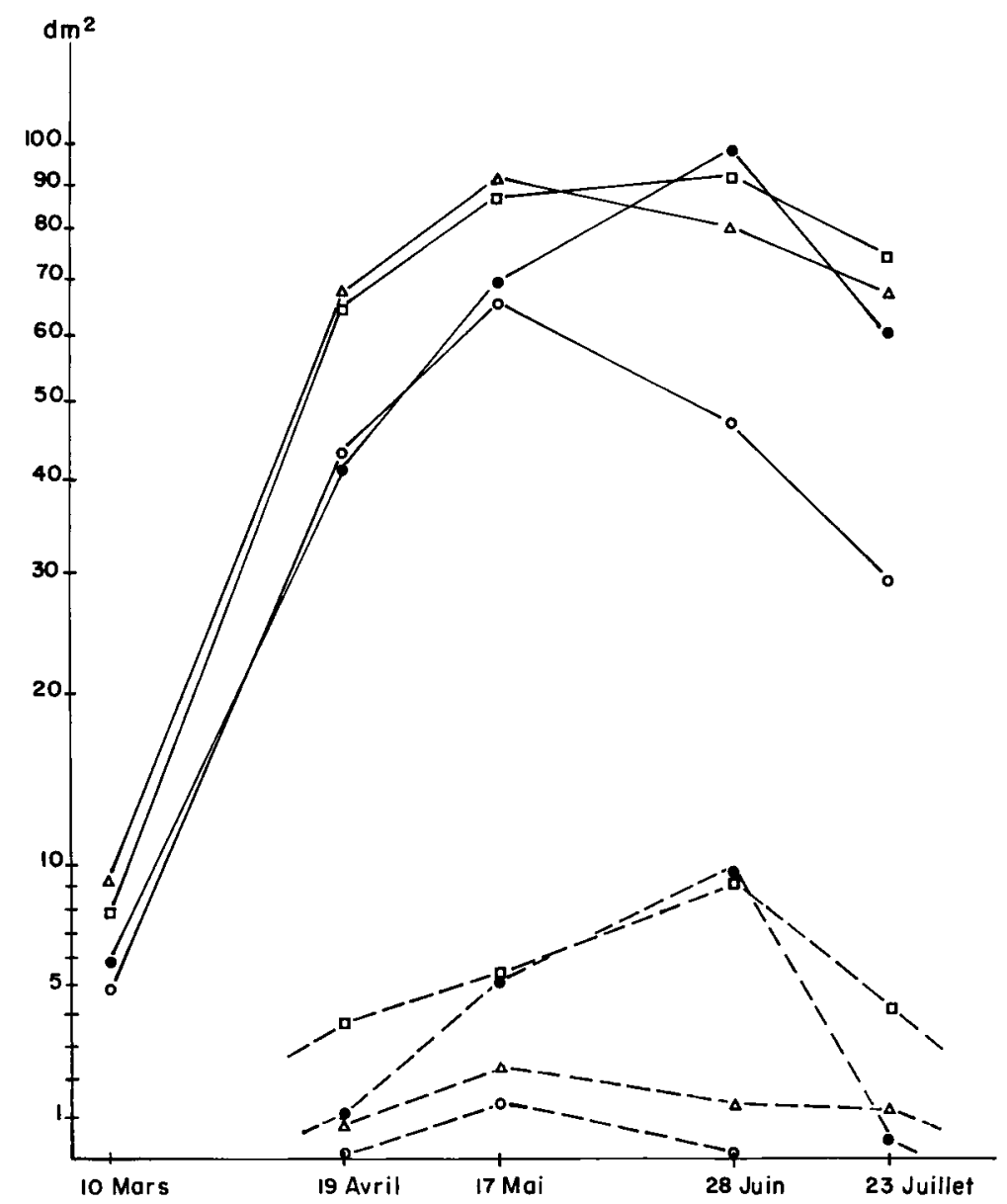

FIG. 1. - Moyennes des surfaces de couvain mesurées au Rheu en 1971

En traits pleins : surfaces totales de couvain. En trait discontinu : surface du couvain de mâles.

$\mathrm{O}=$ Groupe I,

$\triangle=$ Groupe II,

$\square=$ Groupe III,

- Groupe IV.

Aвв. 1. - Mittel der Brutfächen gemessen in Rheu im Jahre 1971

Ausgezogene Kurven : Gesamtbrutfläche.

Gestrichelte Kurven : Drohnenbrutfäche. 
La figure 1, qui concerne l'année 1971, montre que les courbes moyennes de développement du couvain sont décalées les unes par rapport aux autres selon les groupes. Les abeilles du groupe III (Provence) ont un démarrage plus rapide au printemps et le couvain de mâles se développe plus vite que chez les abeilles du groupe IV (Bretagne). En fin de saison les abeilles de Provence poursuivent plus longtemps leur élevage aussi bien pour le couvain d'ouvrières que pour le couvain de mâles. Le calcul statistique montre que les différences entre les groupes III et IV, aussi bien pour le couvain d'ouvrières que pour le couvain de mâles, sont significatives (Tableau 3). On constate donc un certain parallélisme entre l'élevage du couvain d'ouvrières et du couvain de mâles.

Une remarque s'impose en ce qui concerne l'année 1969. Les observations du 20 mai et du 3 juin montrent la disparition à peu près totale du couvain de mâles; elle est consécutive à une période de très mauvais temps. Il est bien connu que l'élevage des mâles est sujet à des fluctuations importantes et que les larves et les nymphes sont facilement détruites par la colonie en cas de disette.

5 - Nous avons tenté d'établir une corrélation entre l'étendue du couvain de mâles et la surface totale du couvain. Une telle coorélation n'existe pas. Il

\begin{tabular}{|c|c|c|c|c|}
\hline $\begin{array}{c}\text { Groupe } \\
\text { Gruppe }\end{array}$ & $\begin{array}{c}1968 \\
\mathrm{~kg}\end{array}$ & $\begin{array}{c}1969 \\
\mathrm{~kg}\end{array}$ & $\begin{array}{c}1970 \\
\mathbf{k g}\end{array}$ & $\begin{array}{c}1971 \\
\mathrm{~kg}\end{array}$ \\
\hline I. Hybrides 1967 & $\begin{array}{c}0,060 \\
(0,8 \%)\end{array}$ & $\begin{array}{c}8,200 \\
(75 \%)\end{array}$ & $\begin{array}{c}14,300 \\
(64 \%)\end{array}$ & $\begin{array}{c}6,000 \\
(51 \%)\end{array}$ \\
\hline II. Hybrides 1968 & - & $\begin{array}{c}3,200 \\
(29 \%)\end{array}$ & $\begin{array}{c}18,000 \\
(81 \%)\end{array}$ & $\begin{array}{c}2,300 \\
(19 \%)\end{array}$ \\
\hline III. Provence 1968 & - & $\begin{array}{c}2,960 \\
(27 \%)\end{array}$ & $\begin{array}{c}15,640 \\
(71 \%)\end{array}$ & $\begin{array}{c}10,300 \\
(87 \%)\end{array}$ \\
\hline IV. Bretagne 1968/70 & $\begin{array}{c}7,760 \\
(100 \%)\end{array}$ & $\begin{array}{c}10,900 \\
(100 \%)\end{array}$ & $\begin{array}{c}22,120 \\
(100 \%)\end{array}$ & $\begin{array}{c}11,300 \\
(100 \%)\end{array}$ \\
\hline V. Provence $(207 \times 315)$ & & & & 19 \\
\hline VI. Hybrides triples & & & & 44 \\
\hline
\end{tabular}

TABL. 6. - Rendements moyens en miel dans les différents groupes de 1968 à 1971

Pour le rucher du Rheu (groupes I à IV) le chiffre entre parenthèses indique le pourcentage de rendement en miel par rapport au témoin (groupe IV $=100 \%$ ). Le rendement est calculé en tenant compte des nourrissements, des récoltes de miel et des différences de poids à la mise en hivernage. Il est exprimé en kg.

TAB. 6. - Mitlerer Honigertrag bei den verschiedenen Gruppen in den Jahren 1968-1971

Für den Bienenstand in Rheu (Gruppe I-IV) bedeutet die eingeklammerte Zahl den Prozentsatz des Honigertrages in Bezug auf die Kontrolle (Gruppe IV $=100 \%$ ). Bei der Berechnung des Ertrages wurden die Fütterungen, die Honigernte und die Gewichtsunterschiede bei der Einwinterung berücksichtigt. Der Ertrag wird in $\mathrm{Kg}$ angegeben. 
est particulièrement caractéristique de constater que deux colonies de même origine, ayant à peu près la même surface de couvain et une reine de même âge peuvent très bien avoir, l'une beaucoup, l'autre pas du tout de couvain de mâles.

- Il était également intéressant de rechercher un lien entre production de couvain de mâles et rendement en miel. Nous n'avons pu mettre en évidence aucune corrélation, ni positive ni négative. Les deux phénomènes sont apparemment sans rapport. Le rendement d'une ruche étant, de toute façon, sous la dépendance d'un très grand nombre de facteurs, il n'y a pas lieu d'être surpris. Tout au plus peut on remarquer que les hybrides du groupe VI, qui ont un élevage de mâles ( normal ), ont un rendement très supérieur à celui des témoins du groupe $\mathrm{V}$ qui ont un élevage de mâles très réduit (Tableau 6). Dans ce cas particulier, tout indique que les colonies du groupe VI ont bénéficié d'une hétérosis très importante mais ce phénomène est étranger à notre problème. Le rendement en miel des abeilles bretonnes (groupe IV) est supérieur à celui des abeilles provençales (groupe III) et pour ces deux groupes la production de mâles est sensiblement la même.

7 - Si l'on considère ce qui se passe après un changement de reine dans les groupes I à III, on constate, généralement, que le taux de production des mâles se trouve peu modifié. Le cas le plus intéressant est certainement celui de la ruche No 32 (groupe I) qui, en 1971, après deux changements de reine, ne produit que $1 \%$ de mâles. On note le même phénomène avec la ruche No 65 du groupe II.

8 - L'étude biométrique des ouvrières et des mâles provenant des reines qui étaient les filles de celles qui furent introduites à l'origine dans les groupes I et II a montré le faible rôle joué par les mâles ligustica au rucher du Rheu. L'absence de production de mâles dans la quasi-totalité des colonies hybridées des groupes I et II, face à la production normale des colonies des groupes III et IV a, évidemment, contribué à accélérer la disparition des caractères ligustica dans l'ensemble de la population du rucher. C'est $d$ 'ailleurs ce phénomène qui a totalement faussé les expériences primitivement prévues et qui devaient porter sur les incidences de l'introduction de gènes étrangers dans une population de race pure.

\section{DISCUSSION}

Les résultats obtenus permettent tout d'abord de confirmer ceux de M. D. Allen $(1958,1963,1965)$ et de Weiss (1962) en ce qui concerne l'importance absolue et relative du couvain de mâles, la variation au cours de l'année, la variation individuelle, l'absence de corrélation entre couvain de mâles et rendement en miel, l'absence de corrélation entre couvain de mâles et couvain 
d'ouvrières. On notera toutefois que les valeurs obtenues à Montfavet, donc sous le climat méditerranéen, avec des hybrides complexes manifestant une très nette hétérosis (groupe VI) sortent nettement des normes valables pour l'Europe occidentale (M. D. Allen) ou pour l'Europe centrale (WEIss) et pour des colonies de race pure, aussi bien pour les surfaces de couvain d'ouvrières que pour les surfaces de couvain de mâles.

L'hypothèse de Gorbakov selon laquelle l'absence de ponte d'œufs non fécondés serait un caractère anatomo-physiologique héréditaire sous la dépendance d'un gène récessif n'explique pas nos résultats. En effet, le phénomène que nous avons mis en évidence ne correspond pas à la description de l'auteur soviétique. Nous ne constatons pas une incapacité totale de la reine à pondre des œufs non fécondés puisque, presque toujours, on trouve quelques $\mathrm{cm}^{2} \mathrm{de}$ couvain de mâles, au moins au cours d'un relevé. D'autre part, les colonies sans mâles que nous avons observées ont essaimé autant que les autres et ne se sont pas distinguées par une production accrue de miel; les ouvrières n'étaient pas plus grosses que la normale. Rappelons que ces différentes caractéristiques ont été attribuées par GorBAKov aux abeilles « sans mâles » qu'il décrit.

Il est donc très possible que les observations de GorвAкov se rapportent à une mutation d'un seul gène récessif alors que, dans notre cas, il semble beaucoup plus vraisemblable qu'on se trouve en présence d'un caractère polygénique puisque nous trouvons tous les intermédiaires entre l'élevage (c normal » et l'absence à peu près totale d'élevage.

Le caractère génétique du phénomène observé paraît bien établi étant donné qu'on retrouve à l'intérieur des groupes comportant des individus apparentés un taux semblable de production de couvain de mâle. Il est très significatif de constater que la souche de Montfavet No 207 utilisée pour former le groupe V a disparu en 1971 faute de mâles. Or, ce groupe V est remarquable par le bas niveau de son élevage de mâles.

Si l'on examine les caractéristiques génétiques de chacun des groupes, on est amené à constater que le caractère c élevage de mâles réduit » est toujours associé à la présence, dans l'ascendance des reines, de parents ayant fait l'objet d'un élevage consanguin. Drescher (1968) a déjà signalé que les souches fortement consanguines produisent peu de mâles; il attribue cette baisse de production au manque de vitalité des souches consanguines. Si la consanguinité était seule en cause on devrait retrouver automatiquement un niveau normal d'élevage lorsque la consanguinité est éliminée par un ou plusieurs croisements avec des souches non apparentées. Or, nous retrouvons le caractère « élevage de mâles réduit » chez des hybrides de races géographiques dont nous connaissons très bien la généalogie et dont nous savons qu'elles ne sont pas consanguines (groupes I et II). Par ailleurs, les colonies des groupes I et II donnent un abondant couvain d'ouvrières et ne présentent aucun signe d'affaiblissement; 
leurs rendements en miel, inférieurs à ceux des abeilles locales de Bretagne s'expliquent par un manque d'adaptation au milieu (Louveaux, 1966).

Bien que les études soient insuffisamment avancées pour donner une explication correcte du mécanisme génétique en cause, il semble bien que la consanguinité constitue un facteur qui favorise l'apparition de colonies présentant le caractère « élevage de mâles réduit ».

Bien des points restent par ailleurs à préciser. Nous ne savons pas si la réduction de l'élevage des mâles dépend de la reine et concerne le fonctionnement du canal de la spermathèque ou bien s'il relève du comportement des ouvrières par l'intermédiaire de la construction des rayons et des soins apportés aux larves. En effet, on peut fort bien imaginer que les aufs non fécondés soient effectivement pondus par la reine en quantités normales mais éliminés par les ouvrières. On sait que les mâles diploïdes sont détruits de cette façon (Woyke, 1963). On peut également invoquer une incapacité physiologique de la reine à évaluer la largeur des cellules dans lesquelles elle pond. Kaniger (1970) a, en effet, montré que la perception au niveau de la première paire de pattes de la reine de la largeur de la cellule dans laquelle elle va pondre conditionne le sexe de l'cuf.

Il resterait encore à discuter l'intérêt pratique d'une sélection portant sur la capacité d'élevage des mâles. Nous pensons qu'il est triple.

1 - L'insémination artificielle nécessite un grand nombre de mâles. En combinant les techniques apicoles simples qui permettent d'augmenter leur production avec la sélection de souches ayant un taux de production de mâles normal ou supérieur à la normale, on pourrait rendre l'insémination artificielle plus aisée.

2 - Ce qui est vrai pour l'insémination artificielle est vrai pour les stations de fécondation naturelle où on a intérêt à saturer les lieux de rassemblement de mâles au moyen de géniteurs sélectionnés.

3 - Inversement, la possibilité d'obtenir des hybrides de races géographiques à forte hétérosis mais ne produisant que très peu ou pas du tout de mâles constituerait une garantie contre l'hybridation incontrôlée des populations locales.

Cette dernière voie paraît particulièrement intéressante, d'une part parce que, les ayant obtenus dans nos élevages, nous savons qu'il est possible de créer des hybrides à faible ou très faible production de mâles, d'autre part parce que le principal obstacle à l'utilisation des hybrides dans les exploitations apicoles reste encore le danger que ces hybrides font courir aux populations locales en disséminant des mâles indésirables.

La production des mâles nécessaires à l'insémination artificielle à partir de reines n'en donnant pratiquement pas pose évidemment un problème qui 
peut d'ailleurs être résolu de différentes façons; on peut envisager de traiter la reine par le froid ou encore d'utiliser la ponte d'une reine vierge âgée ou bien de se servir d'ouvrières pondeuses filles de la reine choisie.

$$
\begin{array}{r}
\text { Reçu pour publication en juin } 1972 . \\
\text { Eingegangen im Juni } 1972 .
\end{array}
$$

\section{ZUSAMMENFASSUNG}

Jedes normale Bienenvolk zieht im Verlaufe seines biologischen Jahreszyklus eine gewisse Zahl von Drohnen auf. Die aus der gemässigten Zone vorliegenden Beobachtungen zeigen, dass die Produktion von Drohnen immer zeitlich begrenzt ist und dass sie von Volk zu Volk stark schwankt, ohne dass man die Gründe für diese Variabilität kennen würde (AlLEN 1958, 1963, 1965; WeIss 1962).

Nach GoRBaKow (1961) sollen bestimmte Königinnen eine anatomisch-physiologische Anomalie aufweisen, die das Ablegen unbefruchteter Eier verhindert; diese Anomalie sei erblich und durch ein rezessives Gen kontrolliert.

Die vorliegenden Beobachtungen sind, zumindest zu Beginn, völlig zufällig entstanden. Die Autoren wollten für das Studium biometrischer Merkmale bei Hybridbienen die Erzeugung von Drohnenbrut in Gang bringen. In bestimmten Völkern erwies sich dies jedoch als unmöglich, denn die Aufzucht von Drohnen war hier praktisch Null. Wir haben uns deshalb entschlos. sen, diese Beobachtungen weiter zu verfolgen und zu versuchen, die Ursachen der extremen Einschränkung der Drohnenaufzucht in bestimmten Völkern aufzufinden.

Diese Beobachtungen wurden in den Jahren 1968-1971 z.T. in Rheu, Bretagne, und z.T. in Montfavet, Provence, durchgeführt. Es wurden 6 Gruppen von Bienenvölkern gebildet :

In der Bretagne :

Gr:ıpe I. Hybridköniginnen (Ligustica $\times$ Mellifica), unkontrolliert gepaart mit Mellifica - Drohnen der Provence. Für die (instrumentelle) Besamung der Ligustica - Königinnen warden Drohnen einer stark ingezüchteten Mellifica-Linie (Nr. 415) benutzt.

Gruppe II. Dieselbe genetische Kombination wie Gruppe I, aber mit anderer Drohnenlinie (Nr. 360) bei der Besamung der Ligustica - Königinnen.

Gruppe III. Königinnen der Rasse Mellifica aus der Provence, Geschwister, aber keine enge Inzucht.

Gruppe $I V$. Mellifica - Königinnen aus der Bretagne, ohne enge Inzucht.

In der Provence:

Gruppe $V$. Königinnen, entstanden aus einer Kreuzung zwischen 2 Linien (Nr. 207 und 315) der Provençalischen Biene (Rasse Mellifica), die jede für sich seit 1963, bzw. seit 1961 in enger Inzucht gehalten worden waren.

Gruppe VI. Dreifachhybriden : Königinnen Caucasica $\times$ Ligustica, begattet von Mellifica - Drohnen, ohne jede Inzucht.

Die Messungen der Brutflächen erfolgten nach der Methode Fresnaye (1962), bei der statistischen Auswertung gelangte der Test H. von Kruskall und Walurs (1952) zur Anwendung.

\section{Resultate}

1. Die grosse Variabilität der Drohnenproduktion bei verschiedenen Völkern konnte bestätigt werden.

2. Die Flächen von"Drohnenbrut und das Verhältnis der Drohnenbrut zur Gesamtbrutfläche entsprechen den Befunden anderer Autoren; bei den Völkern der Gruppe VI jedoch, bei 
denen ein starker Heterosiseffekt festzustellen war, überschritt die Brutmenge sehr stark das Mittel, und zwar sowohl bei der Arbeiter-wie bei der Drohnenbrut; der relative Anteil der Drohnenbrut hatte sich dabei nicht verändert.

3. Die Variabilität der Produktion von Drohnenbrut ist wesentlich grösser zwischen den Gruppen als innerhalb der Gruppen (Tabellen 1 bis 5 ).

4. Die Völker der verschiedenen Gruppen entwickelten sich verschieden entsprechend ihrer Ferkunft (Louveaux 1966); die Kurven der Entwicklung der Brut flächen im Laufe des Jahres 1971 zeigen für die Drohnenbrut eine deutliche Übereinstimmung mit denen für die Arbeiterbrut (Abb. 1).

5. Im allgemeinen besteht keine Korrelation zwischen der Fläche der Drohnenbrut und der Gesamtbrut fläche.

6. Es besteht keinerlei Korrelation, weder positiv noch negativ, zwischen der Produktion von Drohnenbrut und dem Honigertrag (Tab. 6).

7. Wenn man in den Gruppen I und II die Entwicklung nach einem Austausch der Königinnen verfolgt, kommt man zu dem Schluss, dass sich das Ausmass der Drohnenproduktion von Generation zu Generation nur wenig verändert.

8. Durch biometrische Kontrolle wurde festgestellt, dass die reinrassigen Völker, die zusammen mit den Hybridköniginnen der Gruppen I und II auf demselben Bienenstand aufgestellt waren, trotz des Wechsels vieler Königinnen im Laufe von 3 Jahren nur geringfügige Einkreuzungen erfahren hatten. Das ist eine Folge der sehr geringen Drohnenproduktion in den Gruppen I und II.

Mit der Hypothese von Gorbakow (1961) lassen sich unsere Resultate nicht erklären. Denn wir fanden nicht eine völlige Unfähigkeit der Königin zur Ablage unbefruchteter Eier, sondern nur eine mehr oder weniger starke Reduktion der Drohnenbrut. Diese Reduktion erweist sich deutlich als erbliches Merkmal. Es ist eine polygene Grundlage anzunehmen, während man nach der Beschreibung von GoRBaKow eher eine Mutation vermuten muss.

Bei allen unseren Beobachtungen ist das Merkmal « verminderte Drohnenaufzucht » immer dann aufgetreten, wenn bei einem der Vorfahren der Königin enge Inzucht stattgefunden hat. Es scheint daher so zu sein - ohne dass man jetzt schon eine zufriedenstellenden Erklärung geben könnte - dass die Inzucht das Auftreten von Völkern mit geringer Drohnenzahl begünstigt, und zwar sogar dann, wenn die Inzucht durch mehrere Kreuzungen beseitigt ist.

Schliesslich wird das Interesse der Praxis an der Möglichkeit hervorgehoben, durch Selektion entweder eine Verringerung oder eine Steigerung der Drohnenproduktion zu erzielen. Die Erzeugung von komplexen Hybriden, die einen hohen Grad von Heterosis aufweisen, aber keine Drohnen erzeugen, würde einen erheblichen Vorteil bedeuten, da dadurch das Risiko der unkontrollierten Einbringung fremder. Gene in eine lokale Population, die man so weit als möglich rein erhalten möchte, ganz wesentlich verringert wird.

\section{REFÉRENCES BIBLIOGRAPHIQUES}

Allen M. D., 1958. Drone brood in honey bee colonies. J. econ. Ent. 51, 46-48.

ALLen M. D., 1963. Drone production in honey bee colonies. Nature 199 (No 4 895), 789-790.

Allen M. D., 1965. The effect of a plentiful supply of drone comb on colonies of honey bees. $J$. of apic. res. $4,109-118$.

Drescher W., 1968. Élevage et conservation des reines et des mâles. Ann. Abeille, 11, 255-266.

Fresnaye J., 1962. Un appareil pour le calcul rapide des surfaces de couvain dans les ruches. Ann. Abeille, 5, 145-153.

Gonbakov W. K., 1961. Colonies sans mâles - apiculture future (en polonais). Pszczelarstwo, 12, 19-21 (In Apicultural Abstracts, 16, 145). 
Kreniger N., 1970. Uber die Fähigkeit der Bienenkönigin zwischen Arbeiterinnen-und Drohnenzellen zu unterscheiden. Apidologie 1, 115-142.

KRUSKALL et WALLIS, 1952. Use of ranks in one criterion variance analysis. J. amer. statist. Assoc. 47.

Louveaux J., 1966. Les modalités de l'adaptation des abeilles au milieu naturel. Ann. Abeille 9, 323-350.

Michailov A. S., 1927. (In Gorbakov W. K., 1961 -- Apicultural Abstracts, 16, 145).

WEIss K., 1962. Untersuchungen über die Drohnenerzeugung im Bienenvolk. Arch.f. Bienenk. 39, 1-7.

WeIss K., 1969. Drohnen im Natur-und Pflegevolk. Allg. Dtsch. Imkerztg. 3, 100-105, 5, $131-$ $135,6,164-166$.

WoYkE J., 1963. What happens to diploid drone larvae in a honeybee colony. J. of apicult. Res. 2, 73-75. 